\title{
Effects of Single Amino Acid Substitution on the Dissociation of Multiply Charged Multiprotein Complexes in the Gas Phase
}

\author{
Igor Sinelnikov, Elena N. Kitova, and John S. Klassen \\ Department of Chemistry, University of Alberta, Edmonton, Alberta, Canada \\ Glen D. Armstrong \\ Department of Microbiology and Infectious Diseases, University of Calgary, Calgary, Alberta, Canada
}

\begin{abstract}
The effects of amino acid substitutions on the product ion charge distributions for protonated and deprotonated homogeneous and heterogeneous multiprotein complexes in the gas phase are studied using Fourier-transform mass spectrometry and the blackbody infrared radiative dissociation technique. Notably, it is shown that a single amino acid substitution in the leaving subunit can cause a small but measurable change in product ion charge distribution. Evidence that the degree of charge enrichment of the leaving subunit is influenced by the number of strongly basic or acidic residues within the subunit for the protonated and deprotonated complexes, respectively, is reported. (J Am Soc Mass Spectrom 2007, 18, 688-692) (C) 2007 American Society for Mass Spectrometry
\end{abstract}

$\mathrm{T}$ The dissociation of gaseous, multiply charged multiprotein complexes typically proceeds in a highly asymmetric fashion, whereby a single subunit, with a disproportionately large fraction (in terms of its mass) of the total charge, is ejected from the complex [1-3]. There is evidence of rapid proton transfer between subunits within gaseous multiprotein complexes [4-6]. Additionally, increasing the internal energy or charge state of gaseous di- and multimeric protein complexes or increasing the conformational flexibility of the subunits can increase the average charge state of the leaving subunit [4-6]. Based on these experimental findings, as well as theoretical studies $[7,8]$, it has been proposed that the asymmetric product ion charge distribution may result from the unfolding of the leaving subunit in the gas phase and concomitant increase in acidity/basicity [1-8].

Here, we demonstrate, for the first time, that subtle changes in the primary structure of protein subunits (such as single amino acid substitutions) can influence the product ion charge distribution. Specifically, it is shown that the charge asymmetry observed for the dissociation of multiply protonated and deprotonated protein complexes is particularly sensitive to changes in the number of basic and acidic residues contained within the leaving subunit. To evaluate the influence of

Published online January 16, 2007

Address reprint requests to Dr. John S. Klassen, University of Alberta, Department of Chemistry, Edmonton, Alberta, Canada T6G 2G2. E-mail: john.klassen@ualberta.ca acidic and basic residues on the partitioning of charge between the dissociation product ions in the gas phase, blackbody infrared radiative dissociation (BIRD), implemented with a Fourier-transform ion cyclotron resonance mass spectrometer (FT-ICR/MS) equipped with a nanoflow electrospray ionization (nanoES) source, was performed on a series of gaseous protonated and deprotonated homo- and heterogeneous protein complexes. The B subunits of Shiga toxins (Stx) 1 and 2 served as models systems for this study. The B subunits of Stx1 and Stx2 are 60\% homologous. Notably, they differ in their number of basic (Stx1 B: 2xArg, 5xLys, 1xHis; Stx2 B: 1xArg, 6xLys) and acidic residues (Stx1 B: 5xAsp, 3xGlu; Stx2 B: 5xAsp, 5xGlu). According to nanoES-MS performed on aqueous ammonium acetate solutions of the B subunits of Stx1 and Stx2, the B subunits assemble preferentially to the homopentameric $\left(\mathrm{B}_{5}\right)$ complex at subunit concentrations $>50 \mu \mathrm{M}$ [9]. According to analysis of the X-ray crystal structures, the higher-order structures of Stx $1 B_{5}$ and Stx $2 B_{5}$ are nearly identical [10].

\section{Experimental}

\section{Proteins}

The Stx1 B subunit (MW $7688 \mathrm{Da}$ ) and Stx2 B subunit (MW $7815 \mathrm{Da}$ ) were expressed in Escherichia coli using a procedure described previously [11], purified to $>95 \%$ purity by affinity chromatography [12], dialyzed against $50 \mathrm{mM}$ ammonium acetate (pH 7), and stored at 
$-20{ }^{\circ} \mathrm{C}$. The coding sequence of the Stx1 B subunit gene was altered to produce the mutant proteins. The plasmids containing these mutated Stx1 B subunit gene sequences were then transformed into the BL21-AI E. coli expression strain. Single colonies of each transformant were then grown with shaking (200 rpm) in $20 \mathrm{~mL}$ of $\mathrm{LB}_{\text {carb100 }}$ overnight at $37^{\circ} \mathrm{C}$. These cultures were then diluted into $1 \mathrm{~L} \mathrm{LB}_{\text {carb100 }}$ and incubated at $37^{\circ} \mathrm{C}$ with shaking $(200 \mathrm{rpm})$ until an $\mathrm{OD}_{600}$ of between 0.6 and 0.9 was reached. The expression of the mutant Stx1 B subunits was induced by adding $0.2 \%$ L-arabinose to the cultures for $3 \mathrm{~h}$. The cultures were then treated with $0.1 \mathrm{mg} / \mathrm{mL}$ polymixin B sulfate for $30 \mathrm{~min}$ at $37^{\circ} \mathrm{C}$. These polymyxin-treated cultures were centrifuged at $7000 \mathrm{rpm}$ for $10 \mathrm{~min}$ at $4{ }^{\circ} \mathrm{C}$ and the mutated Stx $1 \mathrm{~B}$ subunits were affinity purified from the resulting cellfree culture supernatant solutions using Synsorb-Pk. Purified protein was exchanged in $50 \mathrm{mM}$ aqueous ammonium acetate ( $\mathrm{pH} 7)$ using an Amicon microconcentrator with a molecular weight cutoff of $10 \mathrm{kDa}$. Stock solutions were stored at $-20^{\circ} \mathrm{C}$. NanoES solutions were prepared by thawing the stock solutions at room temperature and diluting an aliquot to a concentration of $10-50 \mu \mathrm{M}$ with aqueous $20 \mathrm{mM}$ ammonium acetate.

\section{Mass Spectrometry}

All experimental measurements were performed using an Apex II 9.4 T FT-ICR/MS (Bruker, Billerica, MA) equipped with a modified external nanoES ion source. NanoES tips, with an o.d. of 4-7 $\mu \mathrm{m}$, were pulled from aluminosilicate tubes ( $1 \mathrm{~mm}$ o.d., $0.68 \mathrm{~mm}$ i.d.) using a P-2000 micropipette puller (Sutter Instruments, Novato, CA). A platinum wire, inserted into the other end of the nanoES tip, was used to establish electrical contact with the nanoES solution. A potential of $\pm 600-800 \mathrm{~V}$ was applied to the platinum wire in the nanoES tip to spray the solution. The tip was positioned 1-2 mm from a stainless steel sampling capillary using a microelectrode holder. Typical solution flow rates were 20 to $50 \mathrm{~nL} /$ min, depending on the diameter of the nanoES tip and the voltage used. Charged droplets and solvated ions emitted by the nanoES tip were introduced into the vacuum chamber of the mass spectrometer through a heated stainless steel sampling capillary $(0.43 \mathrm{~mm}$ i.d. $)$ maintained at an external temperature of $66^{\circ} \mathrm{C}$. The gaseous ions sampled by the capillary $( \pm 48 \mathrm{~V})$ were transmitted through a skimmer $( \pm 4 \mathrm{~V})$ and accumulated for $2-5 \mathrm{~s}$ in an rf hexapole $( \pm 600 \mathrm{~V}$ p-p). The ions were subsequently ejected from the hexapole and injected at $\pm 2700 \mathrm{~V}$ into the bore of the superconducting magnet, decelerated, and introduced into the ion cell. Two flexible heating blankets placed around the portion of the vacuum tube that surrounds the ion cell were used to control the temperature of the ion cell for the BIRD experiments. The typical base pressure for the instrument was about $5 \times 10^{-10} \mathrm{mbar}$. Data acquisition was performed using XMASS software (version 5.0.10).
The time-domain signals, consisting of the sum of 100 transients containing $128 \mathrm{~K}$ data points per transient, were subjected to one zero-fill before Fouriertransformation.

\section{Data Analysis}

The absolute value of the weighted average leaving subunit charge state $(A C S)$ was calculated using the following expression:

$$
A C S=\left|\sum f_{x} n_{x}\right|
$$

where $f_{x}$ and $n_{x}$ represent, respectively, the fractional abundance and charge state number of the leaving subunit ions. $f_{x}$ was calculated from measured intensities of the leaving subunit ions $\left(I_{x}\right)$ using the following expression. Because the ion signal in FT-ICR/MS is proportional to both the abundance and charge state of the ion, the measured intensities were normalized for charge state:

$$
f_{x}=\left(I_{x} / n_{x}\right) /\left(\sum I_{x} / n_{x}\right)
$$

The values of $A C S$ and the corresponding uncertainty were determined from a minimum of five mass spectra, each of which is the sum of 100 individual transients.

\section{Results and Discussion}

Replicate BIRD measurements performed on the $\mathrm{B}_{5}^{-12}$ ions of Stx1 and Stx2 under identical experimental conditions revealed a statistically significant difference in the $\mathrm{B} / \mathrm{B}_{4}$ product ion charge distributions. The absolute value of the weighted average leaving subunit charge state (ACS) of Stx2 is $4.84 \pm 0.02$ compared to $4.52 \pm 0.02$ for Stx1. In contrast, BIRD of the $\mathrm{B}_{5}^{+12}$ ions of Stx 1 and Stx 2 showed that the leaving subunit of Stx $1 B_{5}$ undergoes greater charge enrichment with an ACS of $4.46 \pm 0.01$ compared to $4.15 \pm 0.01$ for Stx $2 B_{5}$ (Figure 1). Notably, within experimental precision, the measured ACS values are independent of the extent of reaction. This implies that the $\mathrm{B} / \mathrm{B}_{4}$ product ions are produced by parallel dissociation pathways from a single reactant. It follows, then, that proton transfer between subunits must be rapid compared to the rate of dissociation, a result consistent with previous experimental studies [4-6]. The differences in charge asymmetry observed for the $B_{5}^{ \pm 12}$ ions of Stx1 and Stx2 are qualitatively consistent with differences in the number of residues with the highest intrinsic gas-phase basicity (Arg, His, Lys) [13] and lowest intrinsic gas-phase acidity (Asp, Glu) [14] within the B subunits of Stx1 and Stx2.

To establish whether specific acidic and basic residues within the protein subunits influence the partitioning of charge between product ions, BIRD was performed on the \pm 12 ions of homogeneous $B_{5}$ complexes 
(a)

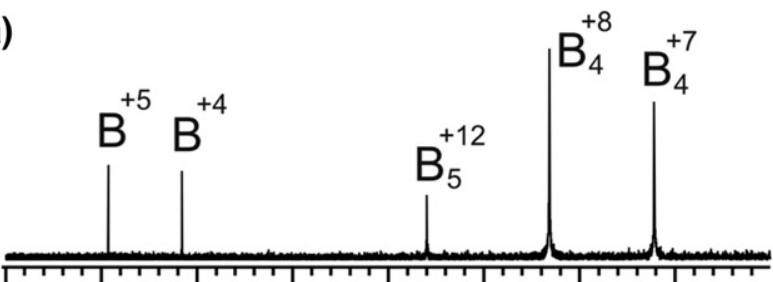

(c)

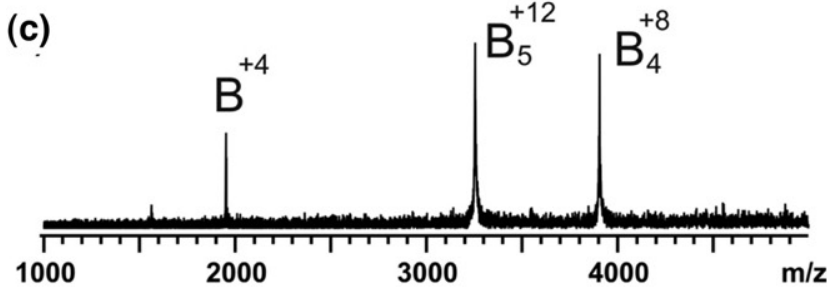

(b)
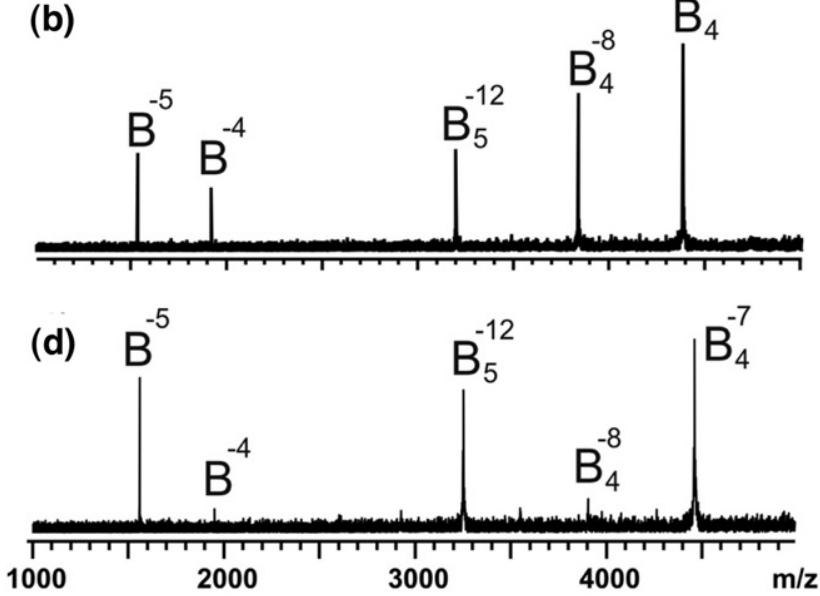

Figure 1. Illustrative BIRD mass spectra obtained at a cell temperature of $160{ }^{\circ} \mathrm{C}$ and a reaction time of $5 \mathrm{~s}$ for the protonated $\mathrm{B}_{5}^{+12}$ ions of (a) Stx1 B subunit, (c) Stx2 B subunit and deprotonated $\mathrm{B}_{5}^{-12}$ ions of (b) Stx1 B subunit and (d) Stx2 B subunit.

composed of single- and double-point mutants of the Stx1 B subunit: Arg69Ala, Arg69Asp, and Arg69Ala/ Lys08Ala. According to the crystal structure of Stx1 $B_{5}$, Arg69 can participate in both intra- and intersubunit interactions, including an ionic interaction with Glu10, whereas Lys08 is involved in an intrasubunit hydrogen bond with Thr49. The Arg33Asp and Lys13Asp mutants were also considered but found not to assemble into homopentamer in aqueous solution. The mutant Phe68Ala was used as a control. (a)

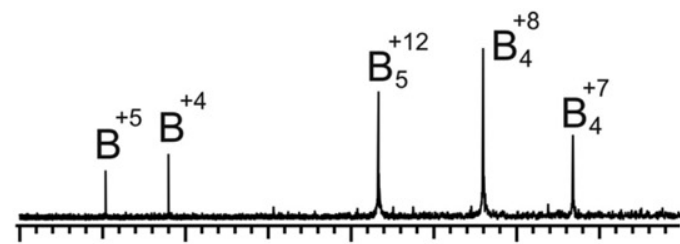

(c)

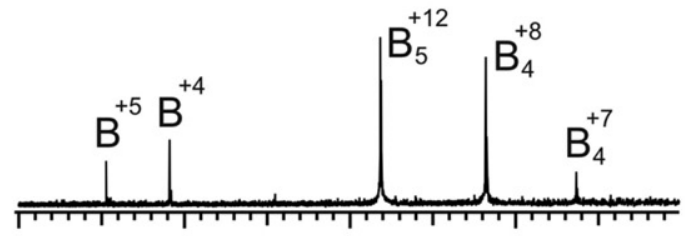

(e)

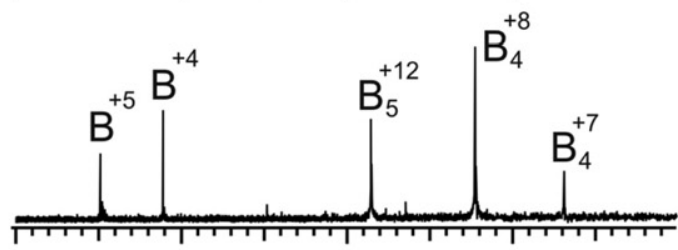

(g)

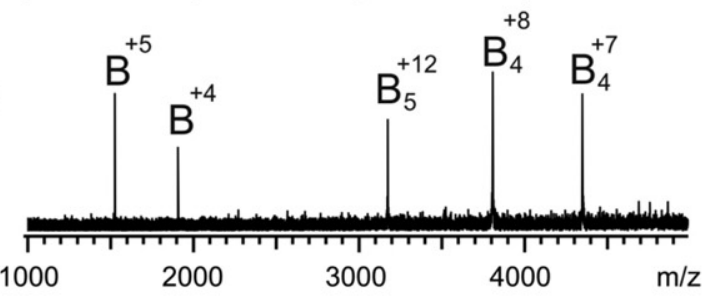

(b)

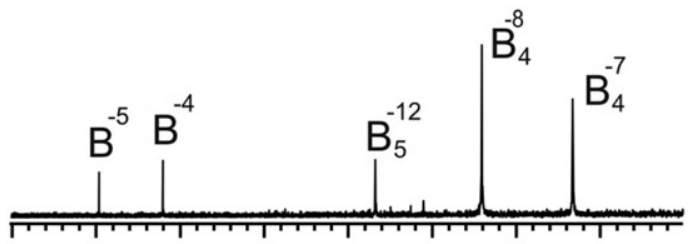

(d)

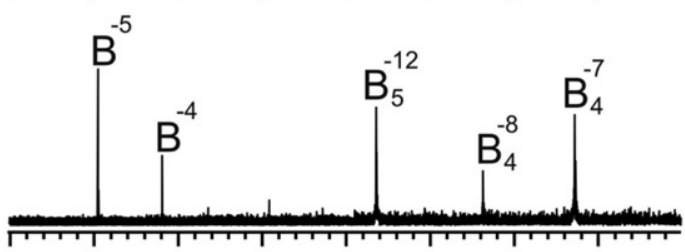

(f)

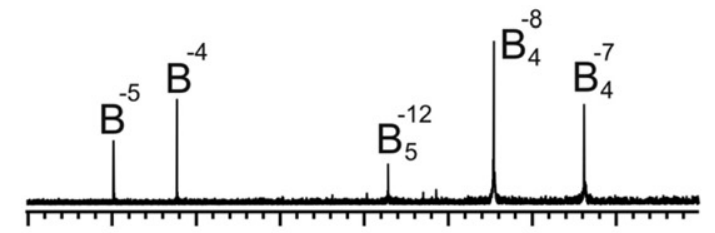

(h)

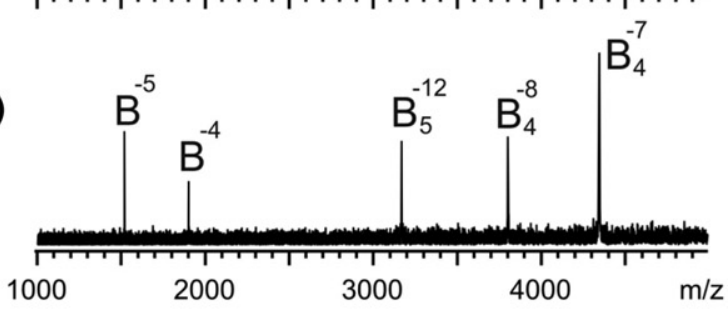

Figure 2. Illustrative BIRD mass spectra obtained for the protonated and deprotonated $B_{5}^{ \pm 12}$ ions composed of (a) mutant Arg69Ala Stx1 B subunit, cell temperature of $149{ }^{\circ} \mathrm{C}$, and a reaction time of $7.5 \mathrm{~s}$; (b) mutant Arg69Ala Stx1 B subunit, $156^{\circ} \mathrm{C}, 5 \mathrm{~s}$; (c) mutant Arg69Asp Stx1 B subunit, $153^{\circ} \mathrm{C}$, $2.5 \mathrm{~s}$; (d) mutant Arg69Asp Stx1 B subunit, $154^{\circ} \mathrm{C}, 15 \mathrm{~s}$; (e) mutant Lys08Ala/Arg69Ala Stx1 B subunit, $154^{\circ} \mathrm{C}$, 2 s; (f) mutant Lys08Ala/Arg69Ala Stx1 B subunit, $154^{\circ} \mathrm{C}, 7 \mathrm{~s}$; (g) mutant Phe68Ala Stx1 B subunit, $158{ }^{\circ} \mathrm{C}$, $3 \mathrm{~s}$; (h) mutant Phe68Ala Stx1 B subunit, $169^{\circ} \mathrm{C}, 1.1 \mathrm{~s}$. 


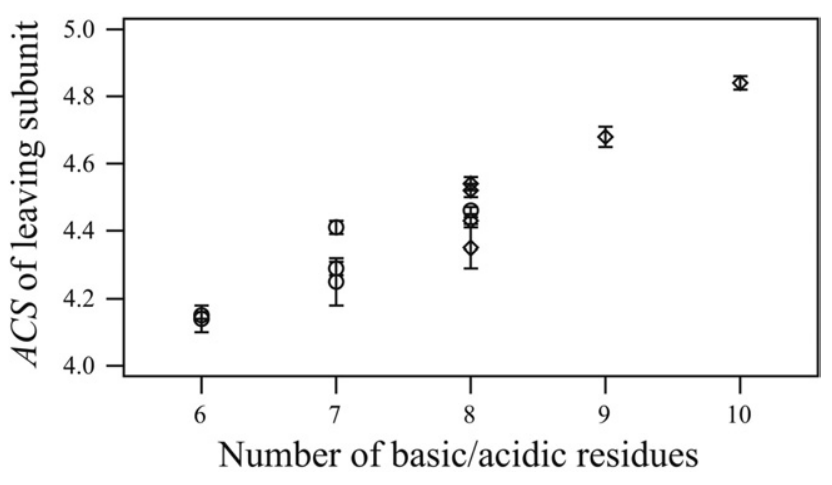

Figure 3. Value of ACS determined for protonated $(\bigcirc)$ and deprotonated $(\diamond) \mathrm{B}_{5}^{ \pm 12}$ ions plotted versus the number of basic (Arg, His, and Lys) or acidic residues (Asp and Glu) contained in subunit of native and mutant Stx1 and native Stx2.

Illustrative BIRD mass spectra of the mutant $B_{5}^{ \pm 12}$ ions are shown in Figure 2. The mutation Arg69Asp results in a small but significant increase in the ACS of the leaving subunit for $\mathrm{B}_{5}^{-12}(\triangle A C S=A C S$ (mutant) $A C S$ (native) $=+0.16 \pm 0.04$ ) but a decrease for Stx1 $\mathrm{B}_{5}^{+12}(-0.17 \pm 0.02)$. The mutation Arg69Ala also results in a decrease in ACS for Stx1 $\mathrm{B}_{5}^{+12}(-0.21 \pm 0.07)$ and, interestingly, for $B_{5}^{-12}(-0.09 \pm 0.02)$. The double mutation, Arg69Ala/Lys08Ala, results in an even greater decrease in charge asymmetry for $\mathrm{B}_{5}^{+12}(-0.32 \pm 0.04)$ and $B_{5}^{-12}(-0.17 \pm 0.06)$. The mutation Phe68Ala resulted in a small increase in ACS for $\mathrm{B}_{5}^{-12}(+0.05 \pm 0.04)$ and for $\mathrm{B}_{5}^{+12}(+0.08 \pm 0.04)$. These results represent the first experimental evidence that changes to individual residues within the subunits of homogeneous protein complexes can influence the product ion charge distribution produced by thermal dissociation in the gas phase. The changes in charge state distribution are admittedly very small, a fraction of a charge state. Nevertheless, they are statistically significant.

In Figure 3, the ACS values, measured for the native and mutant $\mathrm{B}_{5}^{+12}$ ions of Stx1 and Stx2, are plotted versus the number of acidic and basic residues contained within the B subunits. It is evident from the plot that charge enrichment of the protonated and deprotonated leaving subunit of the $\mathrm{B}_{5}^{ \pm 12}$ ions exhibits a degree of correlation with the number of basic and acidic residues, respectively. However, the results obtained for the Phe68Ala mutant reveal that any change in primary structure can influence the ACS.

To establish whether the observed changes in the charge distribution arise from changes in the primary sequence of all the subunits within the complex or just the leaving subunit, BIRD was performed on the -12 ions of the heterogeneous $B_{5}$ complexes composed of native $B$ subunits of Stx1 and Stx2, in all possible combinations, and the +12 ions of the $4: 1$ and $3: 2$ heterogeneous $B_{5}$ complexes composed of native Stx1 B and Arg69Ala B subunits. Shown in Figure 4 are illustrative BIRD mass spectra, acquired for the +12 ions of the $4: 1$ and 3:2 $\mathrm{B}_{5}$ complexes composed of native and (a)

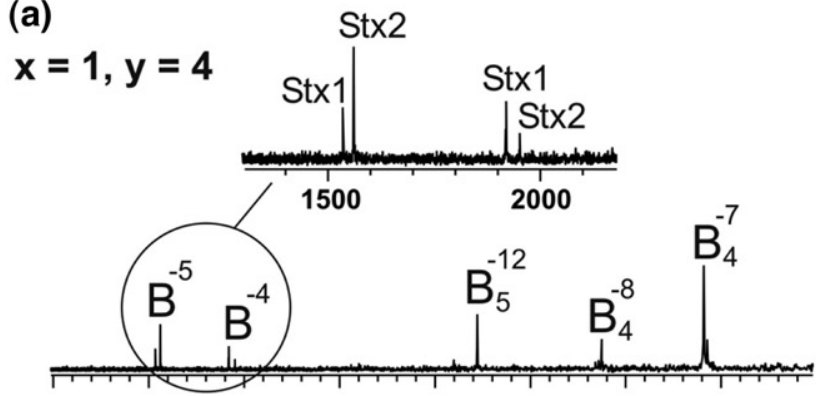

(c)

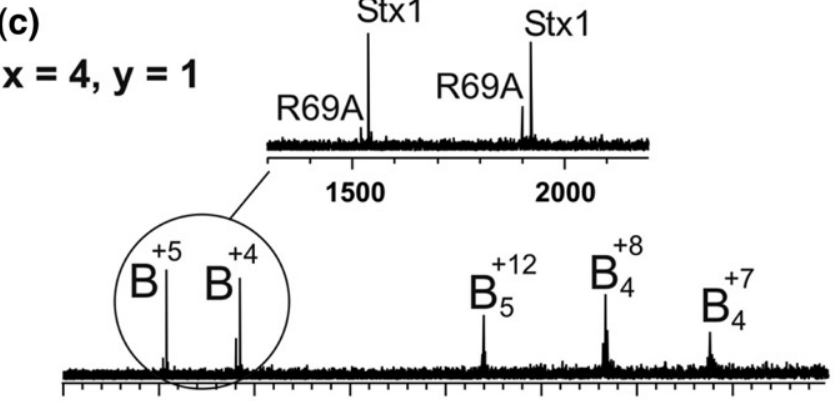

(b)

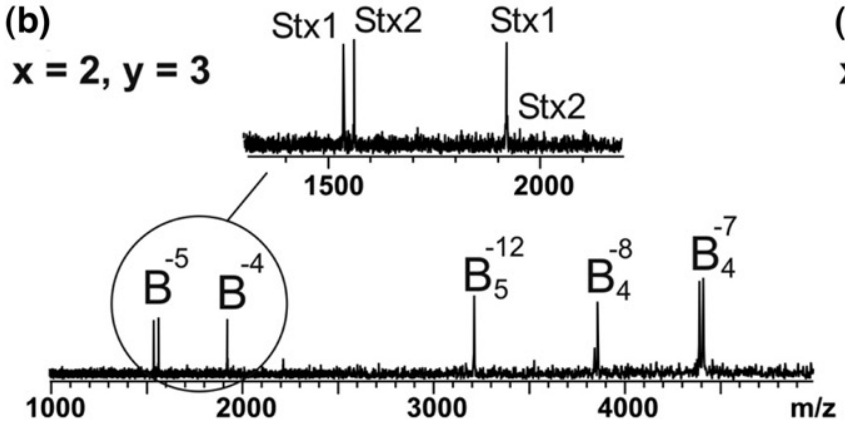

(d)

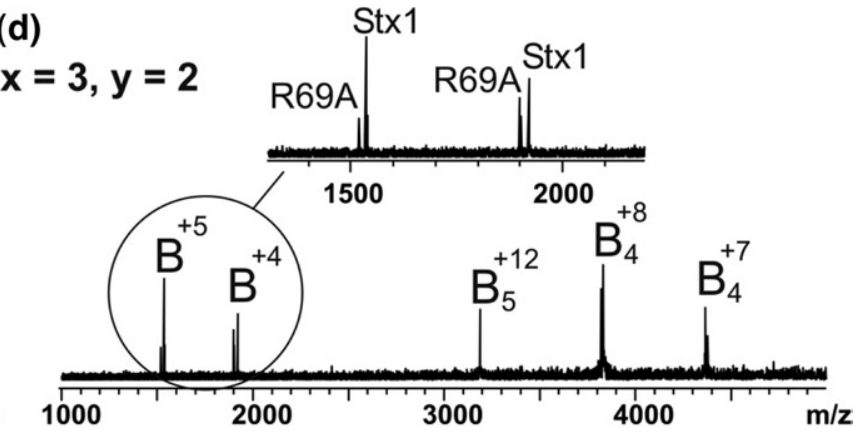

Figure 4. (a, b) BIRD mass spectra obtained at a cell temperature of $166{ }^{\circ} \mathrm{C}$ and a reaction time of $3 \mathrm{~s}$ for the -12 ions of heterogeneous pentamers composed of $\mathbf{x}$ B subunits of Stx1 and $\mathbf{y}$ B subunits of Stx2. (c, d) BIRD mass spectra obtained at a cell temperature of $163{ }^{\circ} \mathrm{C}$ for the +12 ions of heterogeneous pentamers composed of $\mathbf{x}$ native and $\mathbf{y}$ Arg69Ala ( $\equiv$ R69A) mutant B subunits of Stx1, (c) cell temperature of $163^{\circ} \mathrm{C}$, reaction time $5 \mathrm{~s}$, (d) cell temperature of $163{ }^{\circ} \mathrm{C}$, reaction time $2 \mathrm{~s}$. 
Arg69Ala mutant Stx1 B subunits, as well as the -12 ions of the 1:4 and 2:3 $\mathrm{B}_{5}$ complexes composed of native $B$ subunits of Stx 1 and Stx2. For the mixed Stx1B/Stx2B complexes, the ACS values for the loss of Stx1 B (4.47 \pm $0.03)$ and Stx 2 B $(4.78 \pm 0.05)$ were found to be independent of the composition of the complex and identical, within experimental error, to the corresponding values determined for homogeneous pentamers. The $A C S$ values for the loss of native and mutant $B$ subunits from the mixed 4:1 and 3:2 Stx1 B/Arg69Ala B complexes are $4.46 \pm 0.01$ and $4.31 \pm 0.02$, respectively. These ACS values are indistinguishable from the values obtained from the corresponding homogeneous complexes. These results suggest that, for structurally related complexes, the primary sequence of the leaving subunit, independent of the rest of the complex, exerts control over the partitioning of charge during the dissociation process.

The correlation found between the ACS values of the homo- and heterogeneous complexes and the number of acidic (basic) residues within the subunits suggests that addition/deletion of individual acidic (basic) amino acids influences the acidity (basicity) of the leaving subunit, relative to the rest of the complex. The origin of the enhancement may be direct (that is, reflecting the inherent acidity (basicity) of the residue) or indirect in nature, resulting from conformational changes in the leaving subunit (that is, unfolding), which leads to an increase in ACS. For homogeneous complexes, structural differences between the leaving subunit and the remaining subunits would seem necessary to observe a change in ACS upon mutation because the primary structure of each subunit is equally modified. It is also intriguing that a given mutation can have opposite effects on ACS for protonated and deprotonated ions of a given complex. This result suggests that structural changes, if any, imparted by a given mutation are sensitive to the polarity of the ion.

\section{Conclusions}

In summary, we have reported the first experimental evidence that the product ion charge distribution for gaseous protonated and deprotonated homogeneous and heterogeneous multiprotein complexes is sensitive to subtle changes in primary sequence of the leaving subunit. Most significantly, we have shown that a single amino acid substitution within a $38.5 \mathrm{kDa}$ multisubunit complex can alter the product ion charge distribution. For the $\mathrm{B}_{5}^{+12}$ ions composed of the native B subunits of Stx1 and mutant Stx1 B or native Stx2 B it is found that the charge enrichment of the leaving subunit exhibits a degree of correlation with the number of the most acidic/basic residues contained within the subunit. However, evidence that any given mutation can alter the $A C S$ is also reported. These findings are significant in that they argue for the explicit consideration of primary structure in a general theoretical model for the dissociation of gaseous multiprotein complexes. The insights gained in this study may also aid in the interpretation of tandem mass spectra of heterogeneous and structurally related multiprotein complexes.

\section{Acknowledgments}

The authors thank the Natural Sciences and Engineering Research Council of Canada and the Alberta Ingenuity Centre for Carbohydrate Science for financial support and G. Mulvey and T. Dingle for assistance in producing the Stx B subunits.

\section{References}

1. Loo, J. A. Studying Noncovalent Protein Complexes by Electrospray Ionization Mass Spectrometry. Mass Spectrom. Rev. 1997, 16, 1-23.

2. Heck, A. J. R.; van den Heuvel, R. H. H. Investigation of Intact Protein Complexes by Mass Spectrometry. Mass Spectrom. Rev. 2004, 23, $368-$ 389.

3. Benesch, J. L. P.; Robinson, C. V. Mass Spectrometry of Macromolecular Assemblies: Preservation and Dissociation. Curr. Opin. Struct. Biol. 2006, $16,245-251$.

4. Felitsyn, N.; Kitova, E. N.; Klassen, J. S. Thermal Decomposition of a Gaseous Multiprotein Complex Studied by Blackbody Infrared Radiative Dissociation. Investigating the Origin of the Asymmetric Dissociation Behavior. Anal. Chem. 2001, 73, 4647-4661.

5. Jurchen, J. C.; Williams, E. R. Origin of Asymmetric Charge Partitioning in the Dissociation of Gas-phase Protein Homodimers. J. Am. Chem. Soc. 2003, 125, 2817-2826.

6. Jurchen, J. C.; Garcia, D. E.; Williams, E. R. Further Studies on the Origins of Asymmetric Charge Partitioning in Protein Homodimers. J. Am. Soc. Mass Spectrom. 2004, 15, 1408-1415.

7. Schwartz, B. L.; Bruce, J. E.; Anderson, G. A.; Hofstadler, S. A.; Rockwood, A. L.; Smith, R. D.; Chilkoti, A.; Stayton, P. S. Dissociation of Tetrameric Ions of Noncovalent Streptavidin Complexes Formed by Electrospray-Ionization. J. Am. Soc. Mass Spectrom. 1995, $6,459-465$.

8. Csiszar, S.; Thachuk, M. Using Ellipsoids to Model Charge Distributions in Gas Phase Protein Complex ion Dissociation. Can. J. Chem. 2004, 82, 1736-1744.

9. Kitova, E. N.; Daneshfar, R.; Marcato, P.; Mulvey, G. L.; Armstrong, G.; Klassen, J. S. Stability of the Homopentameric B Subunits of Shiga Toxins 1 and 2 in Solution and the Gas Phase as Revealed by Nanoelectrospray Fourier Transform Ion Cyclotron Resonance Mass Spectrometry. J. Am. Soc. Mass Spectrom. 2005, 16, 1957-1968.

10. Fraser, M. E.; Fujinaga, M.; Cherney, M. M.; Melton-Celsa, A. R.; Twiddy, E. M.; O’Brien, A. D.; James, M. N. G. Structure of Shiga Toxin Type 2 (Stx2) from Escherichia coli O157:H7. J. Biol. Chem. 2004, 279, 27511-27517.

11. Marcato, P.; Mulvey, G.; Read, R. J.; Vander Helm, K.; Nation, P. N.; Armstrong, G. D. Immunoprophylactic Potential of Cloned Shiga Toxin 2 B Subunit. J. Infect. Dis. 2001, 183, 435-443.

12. Mulvey, G.; Vanmaele, R.; Mrazek, M.; Cahill, M.; Armstrong, G. D. Affinity Purification of Shiga-like Toxin I and Shiga-like Toxin II. J. Microbiol. Methods 1998, 32, 247-252.

13. Hunter, E. P.; Lias, S. G. Evaluated Gas Phase Basicities and Proton Affinities of Molecules: An Update. Phys. Chem. Ref. Data 1998, 27, 413-656.

14. Bowers, M. T., Ed. Gas-Phase Ion Chemistry, Vol. 2, Academic Press: New York, 1979, Chap. 11. 